\title{
RADIO-EMISSION FROM \\ SUPERNOVAE REMNANTS IN DISTANT GALAXIES
}

\author{
P. NOTNI, H. OLEAK, and G.-M. RICHTER \\ Zentralinstitut für Astrophysik, Potsdam, G.D.R.
}

\begin{abstract}
It is suggested that some 5C2 radio sources previously identified with normal spiral galaxies, are associated with supernova remnants in these galaxies. This hypothesis is tested by the relative radio and optical positions and by luminosity estimates. It could be further tested by possible coincidences of Ohio radio sources with known supernovae, and also by predicted decreases in radio emission.
\end{abstract}

Some sources of the 5C 2 radio survey were identified by G. G. Pooley and S. Kenderdine (1968) with normal spiral galaxies. Exact position measurements on plates of the Schmidt telescope of the Karl Schwarzschild Observatory, Tautenburg, show that the radio sources prefer the region of the spiral arms (Figure 1). In Table I columns 4, 5 and 6 give the observed offsets of the radio positions from the center of each galaxy and the probability (in per cent) that this distance is the result of observational error. The distance modulus (column 8 ) for all but one (5C 2.108) is obtained by adopting a mean absolute magnitude of $-18^{m}$ for the galaxies. For 5C 2.108 Pooley's identification ('blue star') proved to be a supernova of March 1953 in a faint $19^{m} .5$ spiral or
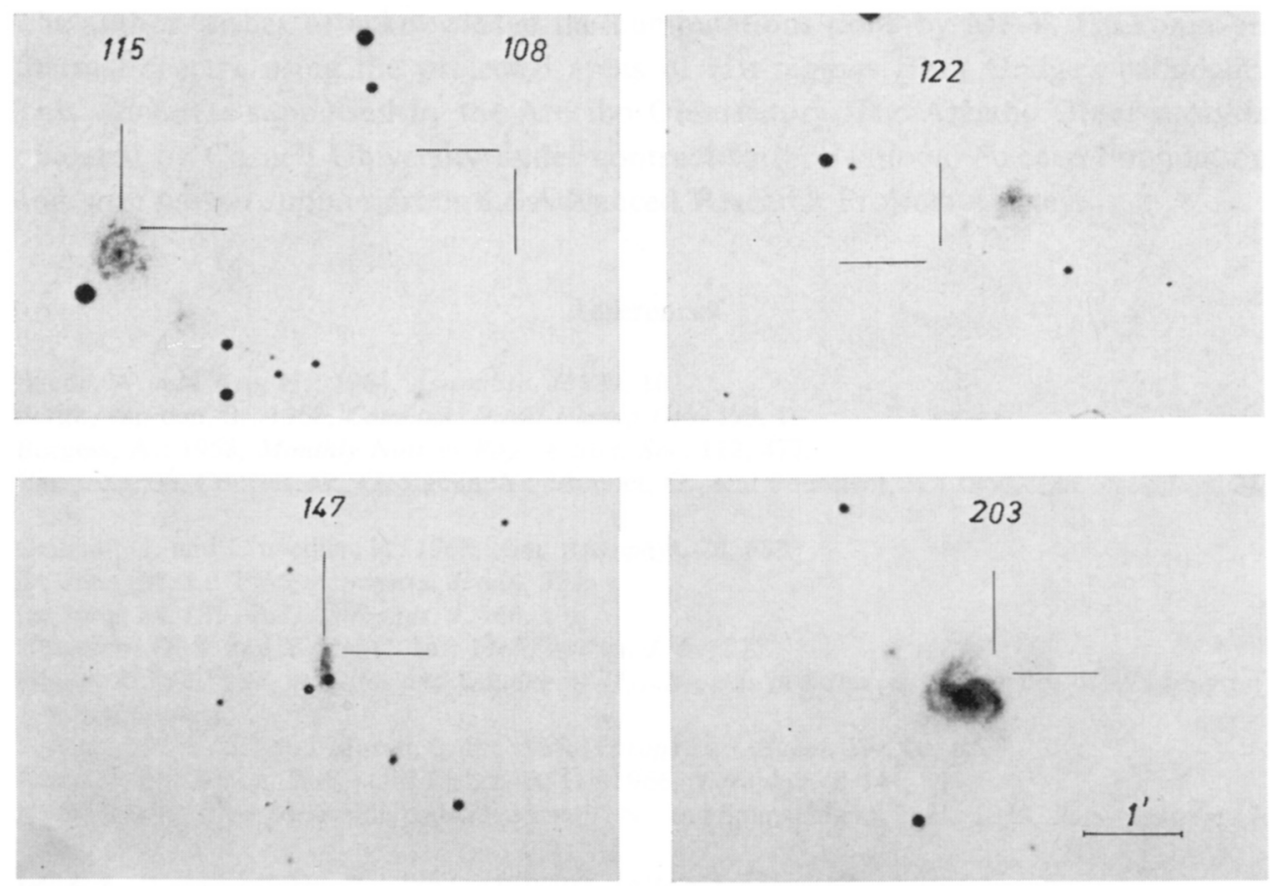

Fig. 1. Radio source positions relative to some spiral galaxies. 


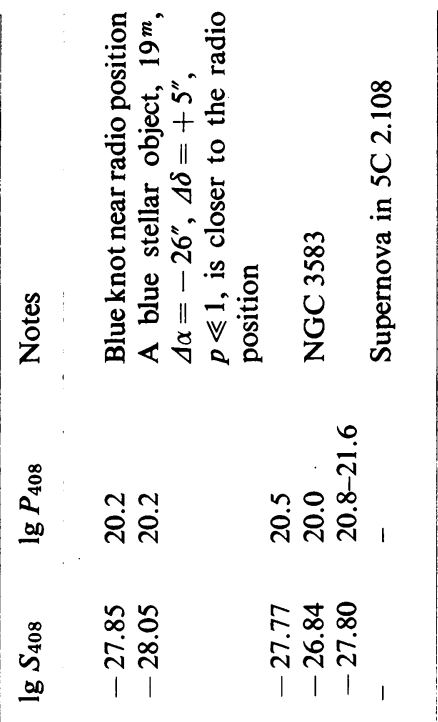

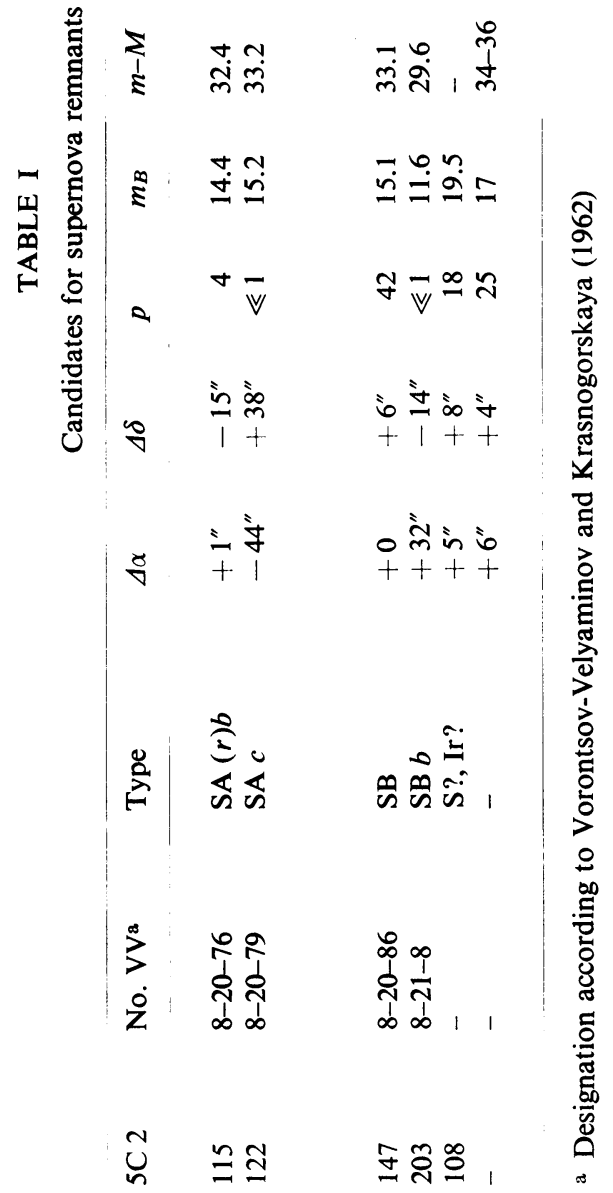


irregular galaxy. Its detection is the reason for including this galaxy in Table I. The supernova has been used to estimate the distance of the parent galaxy which thereby proved to be obviously a system of low luminosity.

The estimated radio luminosity $\left(P_{408}\right.$ in $\mathrm{W} \mathrm{Hz}^{-1}$ ster $\left.^{-1}\right)$ of these galaxies (column 10 ) is of the same order as expected for normal galaxies. Since the radiation obviously does not originate in the nucleus or in the halo as a whole and no optical peculiarities (such as, e.g. jets in weak elliptical radio galaxies) except for knots in the spiral arms are visible, only two possibilities remain for the source of radio emission: H II-regions and supernova remnants. HiI regions can be excluded because of their much lower radio luminosity $\left(<10^{18}\right)$ whereas supernova remnants might possess at some earlier stage of evolution the desired high luminosity of $P \approx 10^{20}$ or $10^{21} \mathrm{~W} \mathrm{~Hz}^{-1}$ ster $^{-1}$. It is

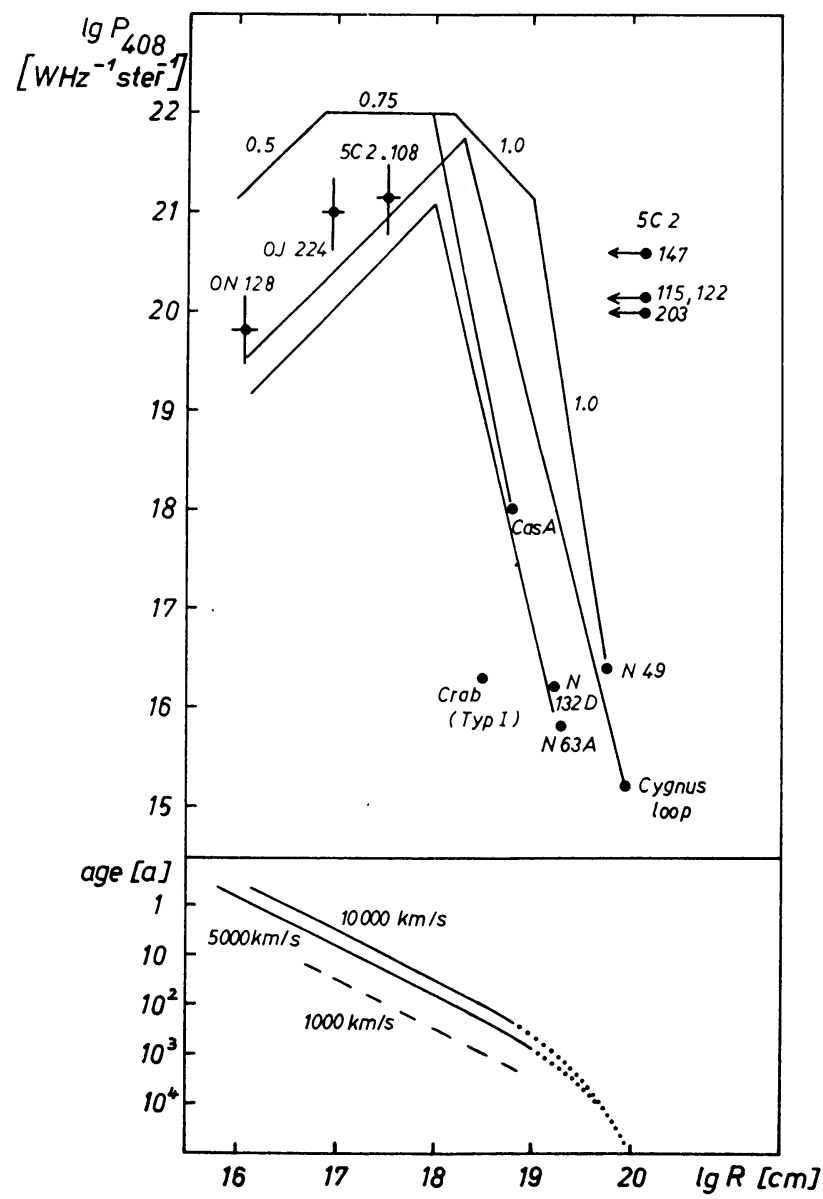

Fig. 2. Extrapolated dependence of radio flux at $408 \mathrm{MHz}$ on source radius for supernovae of Type II. $P \sim R^{5-2 \gamma}$ until the moment at which the source becomes optically thin at decimeter wavelengths, $P \sim R^{-2 \gamma}$ afterwards, where $\gamma=2 \alpha+1$ and $\alpha=$ spectral index. For $\mathbf{N} 49$ the assumed variation of $\alpha$ is marked on the curve. The observed spectral indices are 1.0 (N 49), 0.75 (Cas A), 0.5 (Cygnus loop, N 63 A, N 132D), 0.3 (Crab). The lower part gives the ages for various assumed expansion velocities. 
possible, see e.g. I. S. Shklovsky $(1966, \S 8)$, to extrapolate the observed fluxes of the galactic supernovae backwards to the moment at which the source was optically thick. For spectral indices $\alpha<0.75$ this is the stage of maximum radio luminosity reached about 10 to $100 \mathrm{yr}$ after the outburst. However, the theory must be modified for sources which would reach a flux exceeding about $10^{22} \mathrm{~W} \mathrm{~Hz}^{-1}$ ster $^{-1}$. The total energy-content of the relativistic particles being of the order of $10^{49} \mathrm{erg}$ (Shklovsky $1966, \S 6)$, such a strong radiation of about $10^{22} \mathrm{~W} \mathrm{~Hz}^{-1}$ ster $^{-1}$ implies a maximum lifetime of a few tens of years. In the simple theory the energy loss by radiation is assumed to be negligible, the spectrum remaining constant. If, however, the energy losses amount to a significant proportion of the total energy the spectrum will steepen. Therefore, if all supernovae start with approximately the same low spectral index, e.g. $\alpha=0.5$, the stronger ones, which attain the greatest maximum luminosity should leave the maximum with greater spectral index. Some examples of this behavior are shown in Figure 2, starting from the observed nearby supernova remnants. It can be seen that the radio-maxima of supernovae of Type II probably lie between $10^{20}$ and $10^{22} \mathrm{~W} \mathrm{~Hz}^{-1}$ ster $^{-1}$.

The supernova observed at the position of 5C 2.108 should have had at the time of the radio survey (1966) a radio luminosity of about $10^{21} \mathrm{~W} \mathrm{~Hz}^{-1}$ ster $^{-1}$ lying near its maximum radio brightness. The radio luminosity estimated from the distance modulus 34 to $36 \mathrm{mag}$ (see Table I) is of the same order of magnitude and supports the identification. The other candidates of Table I might also be supernova remnants presumably lying on the descending branch, aged about $10^{2}$ to $10^{3} \mathrm{yr}$.

Stimulated by the above results we looked for radio sources near the positions of known extragalactic supernovae. Among 77 supernovae in regions covered by the Ohio survey (Scheer et al., 1967; Dixon et al., 1968; Fitch et al., 1969) nine coincide with sources on the Ohio maps, two of which are included in the Ohio source list. Only about three coincidences are expected to result by chance. To be conservative we include only the two sources contained in the final Ohio list (Table II). Because of the comparatively low positional accuracy of the Ohio survey, we cannot exclude the possibility in these cases that we are observing the 'normal' radiation of the whole galaxy. As can be seen in Figure 2, ON 128 would lie on the ascending branch and therefore its radio flux should increase during the next decennium by about an order

TABLE II

Suggested identifications of Ohio sources with supernova remnants

\begin{tabular}{|c|c|c|c|c|c|c|c|c|}
\hline No. & NGC & Type & $m_{B}$ & $S N$ & $p^{\prime}$ & $m-M$ & $\lg P_{408}$ & References \\
\hline ON 128 & 4254 & $\mathrm{SA}(s) c$ & 10.5 & $1967 \mathrm{~h}$ & $2 \%$ & 28.5 & 19.8 & $\begin{array}{l}\text { Zwicky: 1968, Publ. } \\
\text { Astron. Soc. Pacific } \\
\text { 80, } 462 .\end{array}$ \\
\hline OJ 224 & Anon. & $\mathrm{SB} c$ & 14.5 & $1962 f$ & $4 \%$ & 32.5 & 21.0 & $\begin{array}{l}\text { Zwicky et al.: } 1963 \text {, } \\
\text { Publ. Astron. Soc. } \\
\text { Pacific 75, } 236 .\end{array}$ \\
\hline
\end{tabular}

$p^{\prime}=$ Probability of chance agreement of positions. 
of magnitude. The apparent diameters of all sources are expected to be very small; about $10^{-4}$ seconds of arc for 5C 2.108, ON 128 and OJ 224.

We expect a strong supernova of Type II to possess a radio luminosity of more than $10^{20} \mathrm{~W} \mathrm{~Hz}^{-1}$ ster $^{-1}$ for about $100 \mathrm{yr}$. This time interval being nearly the rate of occurrence of supernovae in an average spiral, a high percentage of them should emit a radio flux at this level from a 'point' source. This is in accordance with the findings in the 5C 2 survey: three out of six spirals of magnitude 14-15.5 within the central section of the survey (diameter $2^{\circ}$ ) are included in our Table I.

Note added in proof. Because of a subsequently detected systematic error in the radio positions, the observed offsets from the centers of the galaxies are changed by small amounts (Astron. Nachr., in press). The conclusions of the paper remain unaltered. From Table II the source ON 128 must be deleted; it has been found to be an extended source (O. de la Beaujardière et al., Ann. Astrophys. 31, 387, 1968).

\section{References}

Dixon, R. S. and Kraus, J. D.: 1968, Astron. J. 73, 381.

Fitch, L. T., Dixon, R. S., and Kraus, J. D.: 1969, Astron. J. 74, 612.

Pooley, G. G. and Kenderdine, S.: 1968, Monthly Notices Roy. Astron. Soc. 139, 529.

Scheer, D. J. and Kraus, J. D.: 1967, Astron. J. 72, 536.

Shklovsky, I. S.: 1966, in Supernovae, Moscow.

Vorontsov-Velyaminov, B. A. and Krasnogorskaya, A. A.: 1962, Morphological Catalogue of Galaxies, Part I, Trudy gos. Astron. Inst. Sternberga, 32. 